\title{
Risk and Society - the Cultural Foundations of the Brand Image of Insurance
}

\author{
by Orio Giarini*
}

\section{Some basic reasons for the low profile and of the inadequate public perception of insurance in the traditional economic and societal culture}

For decades and decades, the insurance industry has been complaining of the low profile of this branch of economic activity in the public opinion. Many efforts have been made to try to ameliorate the image of insurance, but with limited results.

A recent study made in the United States ${ }^{1}$ indicates that university students still consider the insurance profession as fundamentally "without challenge". Out of ten careers proposed, insurance is only at the ninth place in terms of its prestige. $25 \%$ of all students don't have any idea of what the insurance profession is. Only one student out of 380 from Stanford University entered an insurance company in 1986, seven over three promotions from Harvard, twelve from Columbia University and sixteen from Wharton.

Equally unsatisfactory is the image of insurance concerning the broader public in most countries of the world.

My contention is that this result is not due to the lack of willingness by insurance companies and associations to ameliorate their ways of functioning and meeting their customers needs and the wider public expectations.

The fundamental reasons for such situation are rooted into some key paradigms or basic assumptions of the traditional economic and societal culture which is still strong enough to produce inadequate perceptions of the real importance of insurance and of its risk management role.

Fortunately, these basic assumptions are now submitted to a deep change: because of this process, a new image of insurance is emerging, which should completely revise the traditional attitudes and perceptions within the next decade.

\footnotetext{
* Secretary General of the Geneva Association

1 Mentioned by the ARGUS, Paris, February 5, 1988.
} 
Let us examine one by one these basic assumptions which still permeate our economic and societal culture. They might seem rather theoretical, but it is in fact these basic theories, very often implicit, which mould the key ideas carried by the public opinion as well as by the qualified circles within the economy and the society at large:

- First of all, it should be remembered that economic theory was first crystallized as the consequence of the industrial revolution. It was the industrial development which stimulated and therefore conditioned economic thought. Since Adam Smith, the wealth of nations has been equated with the amount of material goods which the manufacturing system was capable to produce. It is understandable, therefore, that everything not directly connected with the production field, i.e. services (or, in economic terms, the tertiary sector) has been studied with less interest and too easily regarded as a simple auxiliary sector of production. This attitude is still firmly rooted today and is found for example in the extreme lack of accuracy with which services are accounted for in national accounting systems, where the existence of some tertiary activities such as insurance is only partially taken into consideration. Every economic student today still comes out of universities with some knowledge of the Engels' law according to which insurance is bought and used only after some other basic needs (such as food and shelter) are satisfied. And this at a time when the availability of an insurance system is essential, especially in the high technology field, to take more rational decisions for investment and production. The fact that today insurance, as many other service activities, are clearly conditions or factors of production is not yet generally admitted at the level of economic theory and at the level of the general perception, even if it is an easily verifiable fact in practice. The growing perception of the contemporary economy as a service economy will have a decisive impact on the whole economic culture in this respect. This is why the Geneva Association is today so keen to contribute to the world wide discussion on the "new service economy", through "PROGRES" (Research Programme on the Service Economy).

- The second main reason for the rather low relevance of insurance in contemporary economics has been described by Irwing Pfeffer ${ }^{2}$. He shows how the analysis of risk, particularly of insurance risk, requires a dynamic analysis framework, whereas economic theory has chosen in most cases a static method of analysis. The reasons for this are quite understandable if we take as basis the culture and philosophy of the last century. When an expert in the natural sciences analyses the physical or chemical or biological nature of a phenomenon, he can refer to a number of parameters (such as heat, velocity or gravity) which can be identified, isolated, measured and reproduced. This is impossible for the social scientist - no social phenomenon or its constituents can be analysed in the same way as in the natural sciences. He cannot test his hypotheses in a laboratory. If he is an economist, he can only refer to a visible phenomenon such as the price of a product and hence, by logical simulation, reconstruct the forces which resulted in this price at a given moment. Static analysis gives the feeling of precision, of having to deal with determinate systems. As a consequence, economic theory is founded on the notion of "general equilibrium" which excludes uncertainty and risk by definition. Economics has pursued in

\footnotetext{
2 "Perspective on Insurance", edited by Prentice Hall, New Jersey, 1974, and "Insurance and Economic Theory", edited by Irwin, Homewood, Hlinois, 1956.
} 
this way the dream of becoming "scientific" as physics was supposed to be. But since Einstein, even for "hard" sciences, "God appears to play with dice" and, worse still, since Heisenberg, the ultimate physical reality has become "indeterminate". Moreover, when explaining nuclear reactions a physicist once said "it is like managing an insurance company".

We are today at the verge of an important change, where, admitting that the notion of uncertainty or even indetermination are at the core of hard sciences, the dynamic vision of economics, through the integration of uncertainty in its models, is opening the way for a greater interest into the insurance activities which in many ways are prototypes of this new economics of uncertainty.

- The above point is not only relevant as a problem of method to evaluate economic systems in a dynamic rather than in a static way. The whole problem of society facing the uncertainty of life and the basic philosophical assumptions of our culture are here at stake. In fact, behind the dream of a deterministic science aiming at total certainty, which was typical of last century, we simply discover the human dream to fight against the natural uncertainties of the human experience by reference to systems which could provide ultimate certainty. We find a version of this attitude also in the more current economic theory where more and more often reference is made to the notion of "incomplete information". The point is that any information which has to do with events bound to happen in the future is by definition incomplete and can be only considered with reference to various levels of probabilities. No information, when it has to do with real time, is ever complete. It is on the contrary, by definition, uncertain and at best just only highly probable. Human behaviour and even more so economic activities can therefore only be based on decisions taken under different levels of uncertainty. No "scientific" method will ever provide certainty. It is then at this point that the way in which an insurance company sets a premium with consideration to a future probable distribution of events becomes a typical example for managing, in a dynamic way, the contemporary modern economy.

Uncertainty and/or incomplete informations are therefore not at all synonymous of imperfection: they are just a fact of life.

The acceptance of these principles at the level of the roots of societal philosophy depends on the spreading of indeterminist philosophies against the philosophies of determinism $^{3}$.

- The above issues might appear as excessively philosophical and theoretical. But there is an implicit theory behind any human attitude or action. And the complexity of our modern life and economic systems is stimulating our culture to face directly the issues of risk and uncertainty rather than trying to overcome them by a fictious world leaving risk and uncertainty apart.

- At a most practical level, we think that the rising tide of interest in risk management, which has started about twenty years ago, is a clear indication not only of the emergence

3 See for instance Karl Popper: "The Open Universe - An Argument for Indeterminism", Hutchinson, London, 1982. 
of new problems and of new career possibilities, but also of a fundamental change in basic attitudes as to the problems of risk. In any case, key issues discussed today, as for instance the pollution and environmental questions, the vulnerabilities of the industrial systems, the growing concern with liability and other similar matters, are all pointing towards studying and using insurance as a key management tool of present economic realities.

In the next part of this analysis, we shall try to examine closer the new connotations of the contemporary economic system, which we can define as a "service economy". It is in relation with this background that the image of the insurance industry is acquiring a new and quite positive dimension.

\section{The new "service" economy and the strategic role of insurance}

\subsection{The growth of services in the production of wealth}

The growth of service functions is the direct consequence of the development of production technology through the Industrial Revolution. Let us follow it step by step.

Up to the beginning of the 20th century, new technologies and changes in production resulted mostly from improving manufacturing practices on the spot and through work experience. Very rarely were such changes or improvements the consequence of an organised work programme specifically financed in a particular research department or division, inside the company or in a specialised research organisation outside. The professionalisation of research only started during the 1920's, reflecting the growing complexity of new technologies and the need to carefully plan their development and to manage their achievements. This research service function, which developed over the last 60 years, includes today millions of persons and substantial budgets at company and at State level.

Maintenance and storage of incoming raw materials and storage of outgoing products have always been part of even the most simple production processes. But the increasing specialisation of the production units, the more and more complex and advanced technology applied, the growing need to protect the more sophisticated products against damages over increasing transport distances and events of all sorts, have contributed to the continuous increase of the cost of organising service functions throughout the industrialisation process, while the pure costs of production decreased in relative terms.

The distribution of products to more and more people in an increasing number of countries at great distance from production requires the organisation and operation of complex marketing functions without which the product simply cannot reach most of the consumers. The financial activities as well as the insurance functions linked to the performance of production and distribution become essential and ultimately indispensable. When investments for one "machine" such as a nuclear power plant or an oil rig routinely are higher than a billion dollars, the requirement of an adequate functioning of all financial and insurance institutions becomes crucial. 
And as our society becomes more and more complex, so do the regulations governing the interaction of people including product utilisation and safety limitations.

At the beginning of the Industrial Revolution there was little need for a bakery or a textile mill to do any market research in order to define the qualities of its product and to target the market. Today, selling e.g. video-recorders inevitably requires detailed analysis of the potential consumer profiles in terms of regional markets applications, product pricing policy, age groups, etc. . . A variety of liberal professions, from doctors to lawyers, from market researchers and economists to consulting engineers, performs a large number of professional services, either within or attached to the production complex.

Electronic engineers or physicists working in a laboratory clearly have a higher education than the technicians who operated the simple looms used at the beginning of the Industrial Revolution, leave alone the great majority of labour functions performed before the Industrial Revolution which required a very simple level of education. In a pre-industrial society, very few people really could and needed to read whereas in the service society most people will need to have "computer literacy". Mass education has been among the service functions which have passed through a period of rapid expansion, throughout the Industrial Revolution, and today it is a large sector with still great possibilities of improvement. As important and in some cases even larger than the education services in the modern economy are the sectors of health services and national defense.

In order to properly understand and evaluate the modern service economy, it is essential to consider that the growth of services is the result of the specific, successive evolution of the production process itself. The development of technology which changed production

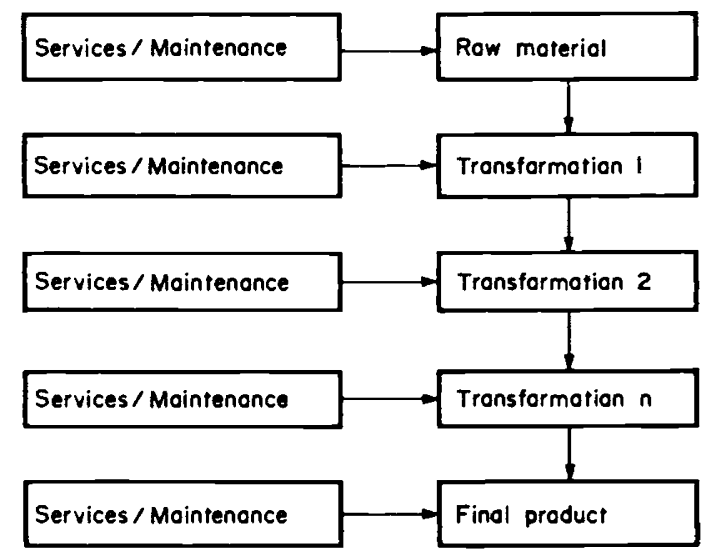

Figure 1: Services and Maintenance in the Production Process

Source: "Dialogue on Wealth and Welfare", by Orio Giarini, Pergamon Press, Oxford, 1980, p. 117. 
processes in order to increase its efficiency, has produced the great development of service functions at all phases of the transformation and utilisation processes.

All the services which we have mentioned are essential in planning and in supporting production up to the point-of-sale. Beyond that, the products themselves need more and more to be managed during their period of utilisation. Further on, the maturing Industrial Revolution has put in evidence another important service: the management of waste.

Waste has always been a by-product of any type of human activity and production: by peeling a banana, we produce waste; by cutting an arrow from a piece of wood, we produce waste. When the Industrial Revolution stimulated a large movement towards the concentration of production and its specialisation, waste inevitably also started to be concentrated and accumulated. This is not necessarily a negative point. During the history of the Industrial Revolution, waste had often been turned into usable by-products and even major new products such as, for instance, the case of nitrogen fertilizers which are often by-products originating from the industry producing explosives, or phosphorous as a base for detergents and fertilizers, stemming from waste produced by the iron and steel industry. In the most advanced stages of the Industrial Revolution, when the principle of the specialisation of products had been stretched to its limits, more and more problems appeared involving wastes which could not economically be transformed into usable products. Furthermore, the fact that more new products were derived from a manipulation of matter benefitting from a deeper knowledge of physics and chemistry, resulted in an increased complexity of waste and a higher level of potential hazards such as poisonning by a greater number of products. Concentration, specialisation and increased levels of dangerous secondary effects are therefore the negative outcome of the use of more sophisticated and advanced sciencebased technology in various sectors. Parallel to the increase of industrial waste, the extension of conspicuous consumption to a constantly increasing number of people has also meant an enormous increase in the amount of waste produced by millions of consumers, in both quantitative and qualitative terms. A plastic bottle cannot always be burned similarly to a piece of wood or paper: it may produce smoke of a corrosive or even poisonous nature. This requires today even more investment to organise an efficient and appropriate disposal system.

Every product finally becomes waste! Most materials, including our bodies, become waste at the end of their production and utilization cycle and some waste is transformed into new raw material. This transformation process happens in some cases naturally, e.g. in the case of organic waste, sometimes only after a delay involving a recycling intervention by man. The recycling of waste is in most cases limited, either by "economic entropy" (when the cost of full recycling would be prohibitive) or by physical (absolute) entropy (when full recycling cannot be done for physical reasons).

Waste handling and disposal is therefore one of the key economic subjects of the service economy. 


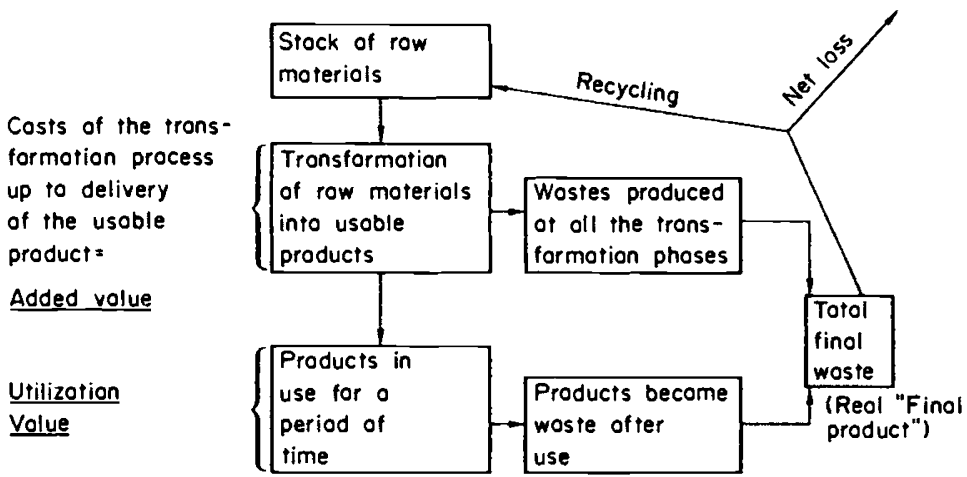

Figure 2: The Real Final Outcome of the Production Process

Source: "Dialogue on Wealth and Welfare", op. cit., p. 31

Figure 2 indicates that, in a situation typical of the Industrial Revolution, the production process was considered as terminated at the moment when a product or a tool were available and sold on the market. In the service economy, the real issue - in terms of economic value - appears to be the maximization of the utilization of products and services together during their life time, taking into account a series of costs which first precede, then accompany production and finally follow it.

On one side, the traditional notion of economic value is linked to the existence and marketability of a product. On the other side, the notion of economic value in the new service economy is extended to include the period of utilization. By consequence, the notion of value in the service economy is in essence not only linked to the factors used in the phase of production, but much more to the value of any product (or service) as looked upon in terms of its performance or result over time. It is the utilisation value during the utilisation period of time which is the point at stake: the effective performance (value) of an automobile as a transport system has to be accounted in terms of its period (and frequency) of utilisation, and the effective benefit (value) of a drug has to be accounted in terms of the level of health achieved. Whereas in the industrial economy the key question was: what is a product's "monetarised" value? the service economy asks another question: what is a product's "utilisation" value; what function does it serve, how well and for how long? The explosion of insurance activities dealing with all forms of liability, of consequential losses, is directly related to this fundamental economic development.

\subsection{The growth of industrialisation within the services/tertiary sector}

The development of the service economy ahead of us has to be considered as a global process of the whole economy following from the Industrial Revolution, rather than simply as the result of growth of the traditional tertiary sector. 
In fact, service functions are integrated into all productive activities in the industrial and also the agricultural sectors. It is essential to notice that modern technology has forced the traditional service or tertiary sector to radically change some of its ways of functioning, by the introduction of processes which are very close to the capital-intensive processes in manufacturing. The distinction between functions performed in a modern computerised office, and a control centre in a production factory is rapidly disappearing. This fact has led some authors, writing about the characteristics of the contemporary economy, to speak about a "super-industrial" economy or a "third Industrial Revolution" instead of speaking of the "service economy". Looking at the most advanced technological sectors, these authors point out that what is in fact happening is a process of industrialisation of the traditional service sectors. ${ }^{4}$ This is clearly an important phenomenon but it overlooks the spectacular increase of service functions within the traditional productive sectors. It would be inadequate to believe that the development of telecommunications, of banking and financial services, of insurance, of maintenance and engineering services, is simply a new kind of "production", an extension of what has happened in the field of textiles, iron and steel and the chemical industry. Selling a shirt (once, in a given moment of time) is a different business to fulfilling a maintenance contract over an extended period of time, during which the seller remains contractually engaged with the consumer for the utilisation of the "product". The point of relevance really concerns understanding what the selling of shirts in a service economy actually means: we switch from an "Industrial Revolution" mentality to a "service economy" mentality, when we add to the costs of their production the costs of maintenance (washing and possibly repairing) during their period of life, plus the costs of their disposal and replacement and we appreciate their value in terms of their actual utilisation.

2.3. The horizontal integration of all productive activities: the end of the theory of the three sectors of economic activity and the limits of Engel's law

Traditional economic theory still distinguishes between three sectors: the primary or agricultural one, the secondary or industrial one, and the tertiary one including all services (sometimes subdivided further in a quaternary one). ${ }^{5}$ This sectorialisation is a "vertical" one and has produced theories of economic development according to which there would be a historical transition from agricultural societies to industrial societies, and there could now be a transition towards a society with a predominant service sector. Such a theory is essentially centered around the industrialisation process, where the predominantly agricultural societies are those which are not yet industrial, and where the tertiary sector very often is simply a "trashcan" used to classify all those economic activities which simply cannot be called industrial.

4 See Irving Leveson, Hudson Institute Strategy Group, New York, "The Service Economy in Economic Development", paper presented at the Graduate Institute of European Studies, Geneva, April 16, 1985.

5 See on this issue Jean Fourastié, "Le grand espoir du XXème siècle", Gallimard, Paris, 1958; Colin Clark, "Les conditions du progrès économique" ("The Conditions of Economic Progress"), PUF, Paris, 1960; Daniel Bell, "The Coming of Post-Industrial Society", Basil Books, New York, 1973. 
In reality, for all three types of society, the agricultural, the industrial and the services one, the relevant point is the reference to the priority to be given to better stimulate the production of wealth and welfare. In an industrial society, agriculture does not disappear, quite the contrary: agricultural production becomes more and more efficient thanks to its industrialisation. Industrialisation does not develop as a completely separate productive activity from agriculture, it also penetrates the traditional way through which agricultural products are produced and distributed. In the same way, the service economy is not the outgrowth of something completely detached from the industrial productive structure, but penetrates the very industrial production which becomes predominantly dependent on the performance of service functions within (as well as outside) the production process. The real phenomenon therefore is not the decline and growth of three vertically separated processes or sectors, but their progressive horizontal interpenetration and integration. In other words, the new service economy does not correspond to the economy of the tertiary sector in the traditional sense, but is built on the fact that service functions are today predominant in all types of economic activities.

A Finnish economist, Pentti Malaska, has expressed this idea in the following graph:

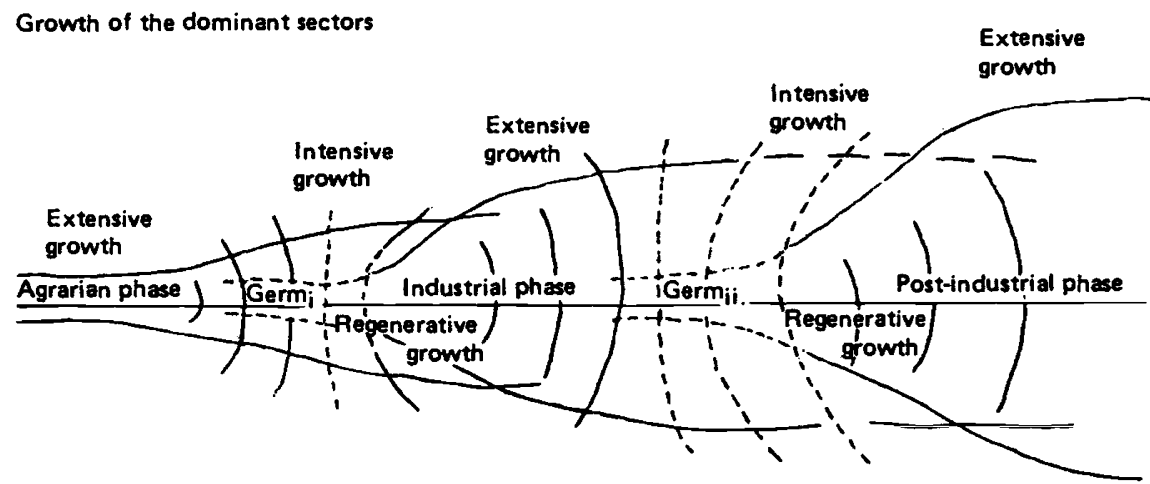

Graph 1: From Pentti Malaska, "Outline of a Policy for the Future", in "The Science of Complexity", the United Nations University, Tokyo, 1985 (page 343).

With every fundamental switch from one priority way to produce wealth and welfare to another, there is a modification in the perception of needs or demand. The definition of what is a basic need also changes.

In an agricultural society, it was obvious that the agricultural (preindustrial) system of production was perceived as facing the problems of satisfying basic needs.

After the start of industrialisation, and in line with the history of economic theory which coincided until now essentially with the development of industrialisation, primary needs have been defined in terms of what basic needs the manufacturing system (integrating 
key agricultural productions) can satisfy. Engel's law states that services are secondary in most of cases, because they only fulfill nonessential needs. The Industrial Revolution, in the same optic, is supposed to be an efficient method to provide food, shelter and health to people. Only once such basic needs are fulfilled, a number of "services" will be consumed.

But the real changes towards the service economy stem precisely from the fact that services are becoming indispensable to make basic products and services fulfilling basic needs available. Services are no longer simply a secondary sector, but they are moving into the focus of the action, where they have become indispensable production tools to satisfy the basic needs and to develop the fundamental tools to increase the wealth of nations.

The insurance industry is a typical example: until a decade ago, everybody, including people in the insurance industry, accepted that insurance policies covering e.g. life risks, or material damages, were a typical secondary product in the traditional economic sense that they could only expand once the basic needs were satisfied by material production. However during the ten years following 1973, when the growth of GNP in the world dropped from an average of $6 \%$ to less than $3 \%$ per year, the overall sales of policies continued to grow about $6 \%$ per year. If insurance consumption were of secondary importance, the slow down in other activities and in particular in manufacturing would have produced more than proportional reduction in the sales of insurance, according to Engel's law.

The explanation for this continuous growth of insurance activities, even in periods of declining growth, lies precisely in the nature of the modern production system which depends on insurance and other services as key tools to guarantee its proper functioning. At a very advanced technological level of production, where risks and vulnerabilities are highly concentrated and represent an essential managerial challenge, insurance has become increasingly so in the last decades - a fundamental precondition for investment. Similarly, at a more general level, social security, health and life insurance have by now achieved the status of a primary need in most industrialised and industrialising countries.

\subsection{From the value of products to the value of systems}

Another key difference between the industrial economy and the service economy is that the first one gives value essentially to products which exist materially and which are exchanged, where value in the service economy is more closely attributed to the performance and real utilisation (in a given period of time) of products (material or not) integrated in a system. Whereas during the classical economic revolution the value of products could be identified essentially with the costs involved in producing it, the notion of value in the service economy is shifting towards the evaluation of costs incurred with reference to obtained results in utilisation. ${ }^{6}$

\footnotetext{
6 There is a far reaching theoretical point here: the rehabilitation of self-production, self-utilisation and selfconsumption (which are by definition non-monetarised activities because outside any direct or implicit exchange system) as key economic resources, which require appropriate management.
} 
The first approach considers the value of a washing machine per se, the second one evaluates the actual performance of the washing machine, taking into consideration not only its cost of production but also all other sorts of costs (learning time of the people using the machine, maintenance and repair costs, etc.). The applicability of the two approaches is inherent in most cases in the technological complexity of the products: in the case of simple products and tools, the assessment of the value can be limited to the tool or the product per se: nobody buying a hammer would think it necessary to take courses to learn how to use it. In the case of a computer, however, the cost of learning how to use it tends to bypass the purchase cost of the machine, especially if the cost of all the necessary software is added.

Similarly, people buying tools such as dishes or even a bicycle will not consider signing a maintenance contract. With purchases of electronic typewriters, photocopying machines, or even television sets, however, maintenance contracts - even for individual consumers are more and more common. In the service economy, the functioning of a tool is being purchased (therefore, including costs of maintenance, repair, and insurance): people are buying functioning systems, not products.

The same type of concept can be found for instance in the health sector. For reasons which are clearly linked to the development of the service economy, institutions called "Health Maintenance Organisations" (HMOs) are growing in importance in the United States, under various forms incorporating the lessons of practical experience.

These organisations combine various elements: incentives to doctors to produce healthy patients rather than big consumers of drugs and hospital services; generalists integrating the collaboration of specialists; the use of new technologies to record all useful data of the patients' medical history; reduced health expenses. The functionings of these HMOs in the United States are very interesting to study in the way they provide better treatment for patients and cost reduction in the health sectors, because the target is an optimal system operation and the value of the HMOs is not identified with the absolute amount of money spent for drugs or hospitalisation. ${ }^{7}$

Money is more efficiently spent when the analysis of the economic value has been swifted to the problem of the performance and results (increasing health) rather than to a pure "industrial" vision (equating more drug consumption with health and wealth increase in all cases).

Systems evaluation, i.e. the organisation of tools and persons in a given environment to obtain desirable and economically valuable results, has then to take into consideration various degrees of complexities as well as the vulnerability in systems functioning. It is in this perspective that the role of insurance becomes a strategically key one in the contemporary service economy.

7 See Alain Enthoven (Stanford University), "The HMO's", lecture presented in Paris, April 23, 1985, at the Institut La Boétie; Martin Zigler, "The Changing Face of Health Care Delivery", in "Emphasis", by Tillinghast Actuaries, March 1985, Atlanta, Georgia.

See also "Problems and Perspectives of Health Insurance", The Geneva Papers on Risk and Insurance No 45 , October 1987, Geneva. 


\subsection{Uncertainty and the vulnerability of systems}

As we have seen, the notion of systems becomes essential in the service economy. Systems produce positive results or economic value when they function properly.

The notion of systems operation (or functioning) requires to consider real time and the dynamics of real life. And whenever real time is taken into consideration, the degree of uncertainty and of probability which conditions any human action becomes a central issue.

The economics of the Industrial Revolution could, in contrast, rely on the fiction of a perfect equilibrium theory (outside real time and duration), based on the assumption of certainty. During most of the economic history of the Industrial Revolution, risk and uncertainty have been an important issue only for historians and sociologists.

Any system working in order to obtain some future results is by definition in a situation of uncertainty, even if different situations are characterised by different degrees of risk, uncertainty or even indetermination. But risk and uncertainty are not a matter of choice: they are simply part of the human condition.

Rationality is therefore not so much a problem of avoiding risks and eliminating uncertainty, but of controlling risks and of reducing uncertainty and indetermination to acceptable and manageable levels in given situations.

Unfortunately, the notion of vulnerability is generally misunderstood. To say that vulnerability increases through the increase of the quality and performance of modern technology might seem paradoxical. In fact, the higher level of performance of most technological advances relies upon a reduction of the margins of error that a system can tolerate without breakdown. Accidents and management mistakes still happen even if less frequently, but their effects have now more costly systemic consequences. Opening the door of a car in motion does not necessarily lead to a catastrophy. In the case of a modern airplane, it will. This shows that the notions of system functioning and of vulnerability control become a key economic function where the contributions of e.g. economists and engineers must be integrated. In a similar way, problems of social security and savings for the individuals have to take into account vulnerability management.

Thus, the notion of risk and the management of vulnerability and uncertainty become key connotations of the service economy.

\subsection{The notion of risk in the Industrial Revolution and in the Service Economy}

The risk taking attitude was not studied as a central theoretical issue by the first great economists: it was rather taken for granted in a given cultural environment, or considered as part of a sociological rather than economic analysis of society. 
Only in 1921 did Frank Knight write a first comprehensive book on the subject of "Risk, Uncertainty and Profit". ${ }^{8}$ But again, the risks that he discussed were more or less limited to the "entrepreneurial" type. The field of the pure risks linked to the vulnerability of systems, was still considered too secondary to be treated as a priority among the managerial objectives of the firm.

Only more recently have economists such as Kenneth Arrow ${ }^{9}$ begun to take a closer look at the reality of the uncertainties that may undermine any economic policy or managerial decision. Just as Ricardo and Smith drew practical examples of their theories from agriculture and small-scale manufacturing, and as the later generation of economists up to Samuelson took their examples from large-scale industry, the most advanced economists of today use the management of risk and uncertainty by insurance institutions and in the social welfare or health sectors as natural reference points.

This has led to a widespread reconsideration of some basic concepts in economic activity, where the fundamental point is the need for a better understanding of the conditions and reasons for modern economic risks and uncertainties that enable the human entrepreneurial talent and creativeness to meet the present challenges in a more successful way. The worldwide discussion on Risk Management is a sign of this process. Basically, it represents reaction to the new nature and dimension of the risks that condition our economic and social environment.

It is clear that risk is today becoming concentrated at levels where the vulnerability is such that the overall uncertainty of the economic process increases. How many Boards of Management today dream of the decision possibilities experienced twenty years ago? Consumers are also reluctant to become increasingly consumers of "risk". The situation in the field of product liability and malpractice in the United States although amplified by a specific legal environment, is more than a localised phenomenon. This is a typical trend of the service economy; the consumer is more and more conscious that tools and products which exist for given purposes and even experts are only of value when the results of their "utilisation" is positive. The fact that their utilisation might give negative results is refuted and gives raise to requests for compensation. Product liability is a great issue in the United States where litigation has led in some cases to extremely high and even excessive compensations. Chemical and pharmaceutical companies have a special problem. ${ }^{10}$ Doctors, lawyers and other experts are sued in court for "malpractice" and have to compensate their clients if found guilty. At the European level, a recent Directive ${ }^{11}$ is the result of ten years of discussions and preparations to manage the expanding phenomenon of the increasing perception by the public that producers of economic wealth have to be liable for delivering a

8 See Frank Knight, "Risk, Uncertainty and Profit", University of Chicago Press, 1971 (1921).

9 See Kenneth Arrow, "Risk Allocation and Information", the Geneva Papers on Risk and Insurance No 8 , June 1978, pp. 5-19, and its bibliography.

10 See the last book from Arthur Hailey, "Strong Medicine", Pan Books, London, 1985.

11 The European Community, Directive on Product Liability, 1985. 
"product" yielding negative results. Once again, in the contemporary economy, it is the "performance" which has economic value, which counts, rather than the simple "existence" of a product or service.

The connotations of the notion of risk in the service economy cover a much larger ground than the notion of risk represented in the Industrial Revolution. In the latter case, the key risk normally referred to is the so-called entrepreneurial or commercial risk; in the service economy, it is extended to the so-called pure risk.

The entrepreneurial risk is one where the people involved in an action can influence its goals and the way the action develops by deciding to produce, to sell, to finance, etc.

The pure risk is out of reach to those involved in an action. It depends on the vulnerabilities of their environment or of the system they are working in, and it will materialise by accident and by hazard. This notion of pure risk is strictly linked to the notion of the vulnerability of systems which we have developed in the preceding paragraphs and its relevance is distinctive of the service economy.

One of the great differences between neoclassical economics and the new service economy is that not only the "entrepreneurial" risk is taken into account (as in the case of Frank Knight), but that the notion of the economically relevant risk is extended to include the notion of pure risk. The notion of risk, globally, has therefore two fundamentally different but complementary connotations.

For any important economic endeavour, the consideration of both notions of risk is today on an equal strategic level (again linked to the notion of systems and of vulnerability).

This means that the management of the pure risk which is largely the object of the insurance, is a key strategic issue for economic development: as a consequence, its image or status is now changing considerably and we should approach in a not too distant future a level of appreciation equal to that of the other sectors of activity which are considered essential for the creation of wealth.

\section{Conclusions}

The conclusions can be very simple:

- On the general cultural level, until the old idea inherited from the 19th century prevails that risk and uncertainty are a kind of imperfection that a pseudoscientific attitude and organisation should eliminate soon or later, it is clear that activities like insurance, managing risk and uncertainty, will always remain a kind of secondary activities, a kind of transient "necessary evil". On the contrary, if the notions of risk and uncertainty are more and more accepted as the normal situation of real life, insurance becomes one of the important sectors developing skills and actions to manage and to overcome the challenges of reality. The transitions of a basic philosophy of society from determinism to indeter- 
minism has therefore as a consequence of transforming the image of insurance from a marginal and secondary one to one of the reference models for our modern world.

- In the same way, at the level of the economy, the traditional theories concerning the creation of wealth exclusively or in priority as a matter of manufacturing systems, lead to consider service activities like insurance as having an ancillary or at best secondary role. On the contrary, the recognition that the creation of economic wealth and welfare depends in the contemporary economy on a blend of manufacturing and service activities, which are both indispensable in the processes of economic development places the insurance activity in the same strategic position as any other important sector of the economy.

Both these two issues (the fundamentals of the philosophy of society and economic theory) are today largely undergoing a profound change and this process is already well advanced even if there is not yet a generalised recognised paradigm which has come to full maturation. But we are close to it.

In any case, this process is accelerated today by the increasing planetary dimension of economic activities and societal and political interdependence. The management of complexity and diversity goes here hand in hand with the problems of the management of uncertainty. ${ }^{12}$

It is also clear that the speed at which this process will be accomplished also depends on the maturation among those responsible for the insurance developments of their new, increased societal role and of the new challenging significance of their profession.

12 On this issue, see "The Limits to Certainty", a report for "The Risk Institute Project", in preparation, by Orio Giarini and Walter Stahel, Geneva, 1988. 